\title{
Temporal Analysis of a Mode-Locked Nd-Glass Laser by Four-Wave Mixing
}

\author{
W. Leupacher and A. Penzkofer \\ Naturwissenschaftliche Fakultät II - Physik, Universität, D-8400 Regensburg, \\ Fed. Rep. Germany
}

Received 5 July 1982/Accepted 20 August 1982

\begin{abstract}
The duration, shape, and background intensity level of pulses of a passively modelocked Nd-glass laser are determined by noncollinear phase-matched four-photon frequency mixing of time-separated fundamental and second-harmonic pulses in a KI crystal. The pulse shape is found to be slightly asymmetric with slower trailing than rising parts. The background intensity level before and behind the main pulse is about $5 \times 10^{-6}$ the peak pulse intensity.
\end{abstract}

PACS: $42.55,42.65,42.80$

The application of mode-locked lasers in time-resolved spectroscopic studies requires an accurate analysis of the temporal behaviour of these lasers. Various techniques have been invented to determine the duration, shape, and background energy level of picosecond lasers. Frequently used methods for the measurement of pulse durations are the two-photon fluorescence, the second harmonic, and the Duguay shutter correlation techniques. The temporal shape and duration are measured with streak cameras. These techniques were reviewed in [1-3]. Since the publication of these reviews further techniques have been developed for the measurement of pulse durations [4-7], pulse shapes $[8]$ and background energy contents $[9,10]$. The shotto-shot pulse fluctuations were analysed in [11].

In this paper we analyse the temporal behaviour of a mode-locked Nd-glass laser by noncollinear phasematched, nonresonant four-photon frequency mixing of its fundamental and second-harmonic pulses. This technique was first applied by Auston [12]. Here the analysis is refined by application of four-photon frequency mixing theory to the determination of pulse duration, shape, and background intensity content. After calibration of the system the duration of picosecond pulses may be measured in single shots.

\section{Method}

The fundamental (frequency $v_{\mathrm{L}}$ ) and second-harmonic (frequency $v_{\mathrm{P}}$ ) wave of a mode-locked Nd-glass laser interact in a nonlinear optical medium by four-photon frequency mixing $v_{\mathrm{P}}+v_{\mathrm{P}}-v_{\mathrm{L}} \rightarrow v_{\mathrm{S}}$. The energy conservation requires $v_{\mathrm{S}}=2 v_{\mathrm{P}}-v_{\mathrm{L}}$. The angle $\varphi$ between the interacting laser beams is adjusted to the phasematching condition $\mathbf{k}_{\mathrm{P}}+\mathbf{k}_{\mathrm{P}}-\mathbf{k}_{\mathrm{L}}=\mathbf{k}_{\mathrm{S}}$. The pump pulses are time separated by an optical delay line and the energy conversion $\eta=W_{\mathrm{S}} / W_{\mathrm{L}}$ of laser light at frequency $v_{\mathrm{L}}$ to frequency $v_{\mathrm{S}}$ is measured versus delay time $t_{\mathrm{D}}$ $\left(t_{\mathrm{D}}=0\right.$ at optimum temporal overlap of pump pulses in sample, $t_{\mathrm{D}}>0$ when pulse at $v_{\mathrm{L}}$ behind pulse at $v_{\mathrm{P}}$ ). The schematic arrangement of the interacting light beams is depicted in Fig. 1.



Fig. 1. Schematic arrangement of noncollinear phase-matched fourwave mixing. (DL: optical delay line; S: interaction medium; L: lens; F : filter; PM: photomultiplier tube) 
The energy conversion $\eta$ is a function of the peak pulse intensity $I_{\mathrm{OP}}$ (frequency $v_{\mathrm{P}}$ ), of the temporal and spatial intensity distributions, the delay time $t_{\mathrm{D}}$, the phasematching angle $\varphi$, the refractive indices $n_{\mathrm{L}}, n_{\mathrm{p}}, n_{\mathrm{S}}$, the absorption coefficients $\alpha_{L}, \alpha_{\mathrm{p}}, \alpha_{\mathrm{S}}$ and the beam divergencies $\Theta_{\mathrm{L}}, \Theta_{\mathrm{P}}$ [13]. The ratio of energy conversion $r\left(t_{\mathrm{D}}\right)=\eta\left(t_{\mathrm{D}}\right) / \eta(0)$ at a fixed pump pulse peak intensity $I_{\mathrm{OP}}$ is given by

$r\left(t_{\mathrm{D}}\right)=\frac{F\left(t_{\mathrm{D}}\right)}{F(0)}$

with [13]

$$
\begin{aligned}
F\left(t_{\mathrm{D}}\right)= & \int_{-\infty}^{\infty} \int_{-\infty}^{\infty}\left\{\int_{0}^{l} h_{\mathrm{P}}\left(\frac{x}{x_{\mathrm{P}}}\right) f_{\mathrm{P}}\left(\frac{t_{\mathrm{P}}^{\prime}}{t_{\mathrm{P}}}\right)\right. \\
& \cdot\left[h_{\mathrm{L}}\left(\frac{\tilde{x}}{x_{\mathrm{L}}}\right) f_{\mathrm{L}}\left(\frac{t_{\mathrm{L}}^{\prime}}{t_{\mathrm{L}}}\right)\right]^{1 / 2} \exp \left[-\alpha_{\mathrm{P}} z-\frac{1}{2} \alpha_{\mathrm{L}} \tilde{z}\right. \\
& \left.\left.-\frac{n_{g \mathrm{~S}} \alpha_{\mathrm{S}}}{2 n_{\mathrm{S}} \cos (\psi)}(\tilde{l}-z)\right] d z\right\}^{2} d x^{\prime} d t^{\prime} .
\end{aligned}
$$

The small effect of the beam divergencies $\Theta_{L}$ and $\Theta_{P}$ on $r\left(t_{\mathrm{D}}\right)$ is neglected. The parameters in (2) are [13]:

$x=x^{\prime}-z \tan \psi, \quad t=t^{\prime}+n_{g \mathrm{~S}}^{2} z /\left(n_{\mathrm{S}} c \cos \psi\right)$,

$\tilde{x}=x \cos \varphi-z \sin \varphi+2 b \sin (\varphi / 2)$,

$t_{\mathrm{P}}^{\prime}=t-n_{g \mathrm{P}} z / c, \quad t_{\mathrm{L}}^{\prime}=t-t_{\mathrm{D}}-t_{0}-n_{g \mathrm{~L}} \tilde{z} / c$,

$t_{0}$ is chosen that $F\left(t_{\mathrm{D}}=0\right)$ is maximal,

$\tilde{z}=z \cos \varphi+x \sin \varphi-2 b \tan (\varphi / 2) \sin (\varphi / 2)$,

$b=l / 2$ (pump pulses overlap in center of sample),

$\tilde{l}=l / \cos (\varphi / 2)$,

$\varphi=\arccos \left\{\left[\left(2 n_{\mathrm{P}} v_{\mathrm{P}}\right)^{2}+\left(n_{\mathrm{L}} v_{\mathrm{L}}\right)^{2}\right.\right.$

$$
\left.\left.-\left(n_{\mathbf{S}} v_{\mathbf{S}}\right)^{2}\right] /\left(4 n_{\mathrm{P}} n_{\mathbf{L}} v_{\mathrm{P}} v_{\mathbf{L}}\right)\right\}
$$

$\psi=\arcsin \left\{n_{\mathrm{L}} v_{\mathrm{L}} \sin \varphi /\left[\left(n_{\mathrm{L}} v_{\mathrm{L}}\right)^{2}\right.\right.$

$$
\left.\left.+\left(2 n_{\mathrm{P}} v_{\mathrm{P}}\right)^{2}-4 n_{\mathrm{L}} n_{\mathrm{P}} v_{\mathrm{L}} v_{\mathrm{P}} \cos \varphi\right]^{1 / 2}\right\} .
$$

$l$ is the sample length; $n_{g \mathrm{~L}}, n_{g \mathrm{P}}, n_{g \mathrm{~S}}$ are the group refractive indices; $c$ is the vacuum light velocity.

The temporal analysis of laser pulses at frequency $v_{L}$ is achieved by comparing calculated energy conversion ratios $r$ with measured values. The spatial pulse shape is assumed to be Gaussian, i.e. $h_{\mathrm{L}}\left(\tilde{x} / x_{\mathrm{L}}\right)=\exp \left(-\tilde{x}^{2} / x_{\mathrm{L}}^{2}\right)$, $h_{\mathrm{P}}\left(x / x_{\mathrm{P}}\right)=\exp \left(-x^{2} / x_{\mathrm{P}}^{2}\right)$. The temporal pulse shape is choosen in the form of $(i=\mathrm{L}, \mathrm{P})$ :

$$
\begin{aligned}
f_{i}\left(t_{i}^{\prime} / t_{i}\right)= & \exp \left(-t_{i}^{\prime 2} / t_{i}^{2}\right)\left[1-\Theta\left(t_{i}^{\prime}\right)\right] \\
& +\left\{\cosh \left[t_{i}^{\prime} /\left(\kappa_{i} t_{i}\right)\right]\right\}^{-2} \Theta\left(t_{i}^{\prime}\right) \\
& +\delta_{i}\left[\Theta\left(t_{i}^{\prime}+T / 2\right)-\Theta\left(t_{i}^{\prime}-T / 2\right)\right] .
\end{aligned}
$$

$\Theta\left(t_{i}^{\prime}\right)$ is a step function $\left[\Theta\left(t_{i}^{\prime}\right)=0\right.$ for $t_{i}^{\prime}<0, \Theta\left(t_{i}^{\prime}\right)=1$ for $\left.t_{i}^{\prime} \geqq 0\right] . \kappa_{i}$ is a fitting parameter for the pulse asymmetry and $\delta_{i}$ represents the background intensity level. $T$ is the round-trip time of pulses in the laser resonator $(T=10 \mathrm{~ns}) . \kappa_{\mathrm{L}}$ and $\kappa_{\mathrm{P}}$ are set equal to $\kappa$ in the calculations.

In case of collinear four-photon frequency mixing of pump pulses at frequencies $v_{\mathrm{P}}$ and $v_{\mathrm{L}}$ in dispersionless and nonabsorbing media the energy conversion ratio $r\left(t_{\mathrm{D}}\right)$ would simplify to

$$
\begin{aligned}
r\left(t_{\mathrm{D}}, \varphi=0\right)= & G\left(t_{\mathrm{D}}\right) \\
& =\frac{\int_{-\infty}^{\infty} f_{\mathrm{P}}^{2}\left(t / t_{\mathrm{P}}\right) f_{\mathrm{L}}\left[\left(t-t_{\mathrm{D}}\right) / t_{\mathrm{L}}\right] d t}{\int_{-\infty}^{\infty} f_{\mathrm{P}}^{2}\left(t / t_{\mathrm{P}}\right) f_{\mathbf{L}}\left(t / t_{\mathrm{L}}\right) d t} .
\end{aligned}
$$

This correlation function was used in [12] for the pulse analysis. The deviation of $r\left(t_{\mathrm{D}}, \varphi \neq 0\right)$ from $G\left(t_{\mathrm{D}}\right)$ increases with phase-matching angle $\varphi$.

\section{Experimental}

The laser system consists of a passively mode-locked Nd-phosphate glass laser (laser glass Hoya LHG5, bleachable dye Eastman 9860). A single pulse is selected from the pulse train by a laser triggered Kerr shutter (opening time: $10 \mathrm{~ns}$ ) and increased in energy by a laser amplifier. The second-harmonic pulse is generated in a KDP crystal of $1 \mathrm{~cm}$ length. The fundamental and second-harmonic pulses are separated with a harmonic beam splitter and directed to the nonlinear medium under the phase-matching angle. In the path of the fundamental laser beam an optical delay line is inserted. A KI crystal of $2 \mathrm{~cm}$ length is used as four-photon mixing sample (phasematching angle in sample $\varphi=25.42^{\circ}$ ). Other nonlinear optical media might be used as well (e.g. water with $\varphi=10.68^{\circ}$ ). The nonlinear susceptibility of the medium has to respond instantaneously to the interacting beams. The generated light at frequency $v_{\mathrm{S}}$ is detected with a photomultiplier tube. The geometrical arrangement of the light beams is depicted in Fig. 1. (For more details see $[13,14]$.)

\section{Results}

The energy conversion $\eta$ is measured versus delay time $t_{\mathrm{D}}$ in the range between $-300 \mathrm{ps}$ and $1 \mathrm{~ns}$. The energy conversions at different pump pulse peak intensities $I_{\mathrm{OP}}$ around $2 \times 10^{9} \mathrm{~W} / \mathrm{cm}^{2}$ are normalized to a fixed peak intensity $\bar{I}_{\mathrm{OP}}$ by using the proportionality $\eta \propto I_{\mathrm{OP}}^{2}$, i.e. $\eta\left(\bar{I}_{\mathrm{OP}}\right)=\eta\left(I_{\mathrm{OP}}\right) \bar{I}_{\mathrm{OP}}^{2} / I_{\mathrm{OP}}^{2}$. The measured conversion ratios $r\left(t_{\mathrm{D}}\right)$ are shown as data points in Fig. 2. The solid curves are calculated by use of (1)-(3). The KI parameters are [13]: $n_{\mathrm{L}}=1.6358, n_{\mathrm{P}}=1.6778, n_{\mathrm{S}}$ $=1.7625, n_{g \mathrm{~L}}=1.6624, n_{g \mathrm{P}}=1.8067, n_{g \mathrm{~S}}=2.2345, \alpha_{\mathrm{L}}$ 


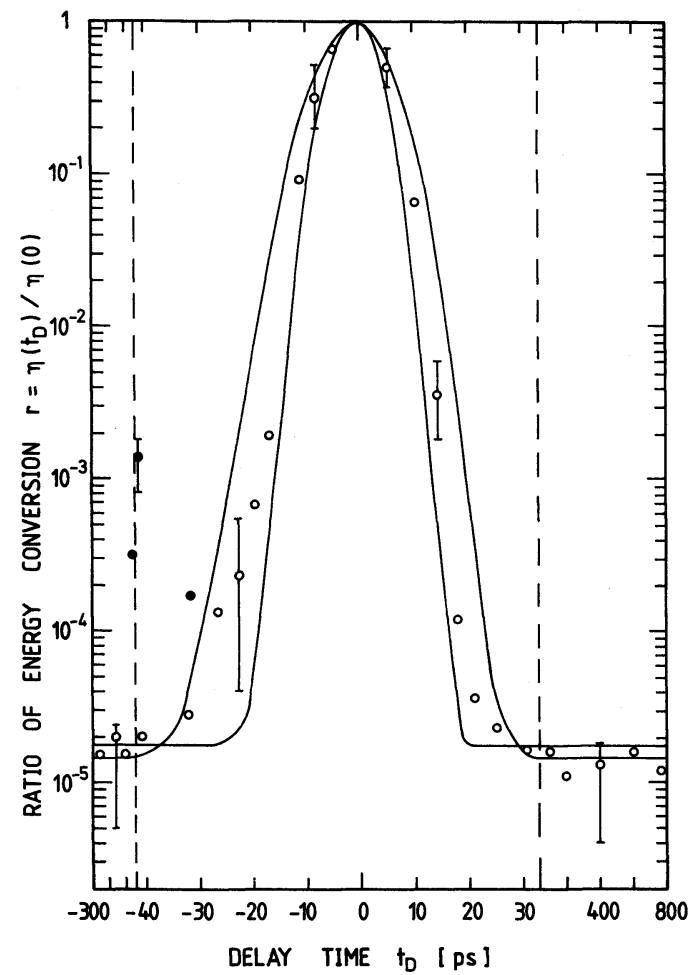

Fig. 2. Ratio of energy conversion versus delay time in $\mathrm{KI}(l=2 \mathrm{~cm})$. Open circles, measured average energy conversions, closed circles indicate influence of reflection of laser at frequency $v_{L}$ from an optical surface. Curves are calculated for rising Gaussian and decaying hyperbolic secant pulses (3) with $\kappa=1.6, \delta_{\mathrm{L}}=5 \times 10^{-6}, \delta_{\mathrm{P}}$ $=\delta_{\mathrm{L}}^{2}, \Delta t_{\mathrm{L}} / \Delta t_{\mathrm{P}}=1.2, \Delta t_{\mathrm{L}}=3 \mathrm{ps}$ (inner curve, FWHM) and $\Delta t_{\mathrm{L}}=6 \mathrm{ps}$ (outer curve). Note change of time scale at dashed vertical lines

$=0.05 \mathrm{~cm}^{-1}, \alpha_{\mathrm{P}}=0, \alpha_{\mathrm{S}}=0.02 \mathrm{~cm}^{-1}$. The laser parameters are $v_{\mathrm{L}}=9480 \mathrm{~cm}^{-1}, \quad v_{\mathrm{P}}=18,960 \mathrm{~cm}^{-1}, \quad v_{\mathrm{S}}$ $=28,440 \mathrm{~cm}^{-1}, x_{\mathrm{L}}=4.2 \mathrm{~mm}, x_{\mathrm{P}}=2.85 \mathrm{~mm}$ and $\Delta t_{\mathrm{L}} / \Delta t_{\mathrm{P}}$ $=1.2$. The pulse durations (FWHM) are $\Delta t_{\mathrm{L}}=3 \mathrm{ps}$ (inner curve) and $6 \mathrm{ps}$ (outer curve). The shape fitting parameter is $\kappa=1.6$, the background to peak pulse intensity ratio is $\delta_{\mathrm{L}}=5 \times 10^{-6}$ and $\delta_{\mathrm{P}}=\delta_{\mathrm{L}}^{2}$.

Second-harmonic generation at small conversion efficiency in dispersionless media would produce pulses of duration $\Delta t_{\mathrm{P}}=2^{-1 / 2} \Delta t_{\mathrm{L}}$ [15-18]. For our situation the ratio $\Delta t_{\mathrm{L}} / \Delta t_{\mathrm{p}}=1.2$ was determined earlier [8].

The experimental points (open circles) fit well calculations for $\Delta t_{\mathrm{L}}=5 \mathrm{ps}, \kappa=1.6$ and $\delta_{\mathrm{L}}=5 \times 10^{-6}$. Each point represents an arithmetic average over 16 shots. The shot-to-shot fluctuations are indicated at some data points by bars which span the region from the forth lowest to the forth highest shot. It should be noted that reflections of the fundamental laser beam (frequency $v_{\mathrm{L}}$ ) at surfaces clearly show up in the postpulse region $\left(t_{\mathrm{D}}<0\right)$ when no antireflection coated or wedged optical components in the path are used. The full circles in Fig. 2 resulted from reflections at an uncoated cylindrical lens.

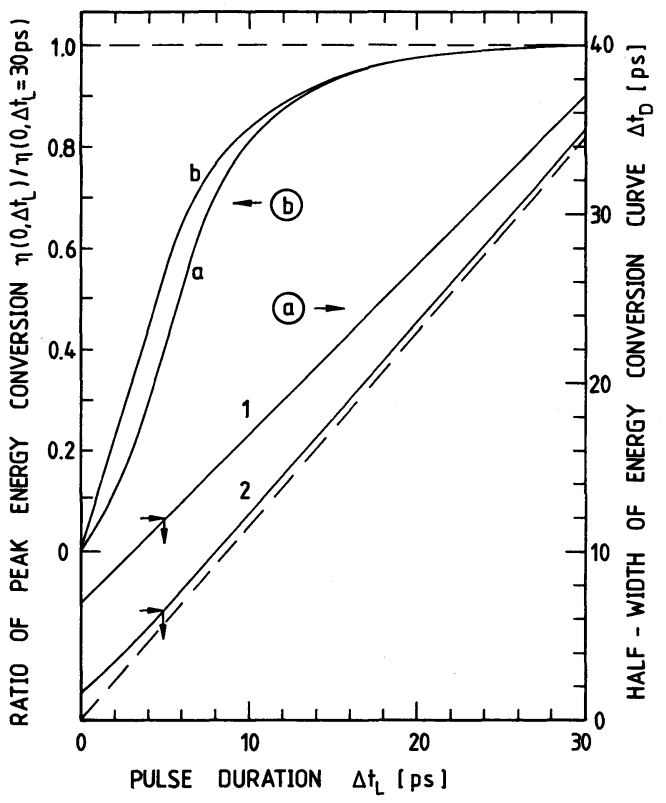

Fig. 3. (a) Calculated half-width of energy conversion curve versus pulse duration. Curve 1 : nonlinear medium is $\mathrm{KI}$; Curve 2: sample is $\mathrm{H}_{2} \mathrm{O}$; dashed curve is responsible for collinear interaction, (4). (b) Dependence of energy conversion on pulse duration. Interaction in $\mathrm{KI}$ (Curve $a$ ), in $\mathrm{H}_{2} \mathrm{O}$ (Curve $b$ ). Dashed curve is valid for collinear phase-matched interaction. Pulse parameters as in Fig. 2 (curves nearly independent of asymmetry parameters $\kappa$ )

The temporal half-width $\Delta t_{\mathrm{D}}$ of $r\left(t_{\mathrm{D}}\right)$ versus pulse duration $\Delta t_{\mathrm{L}}$ is shown in Fig. 3a. Curve 1 indicates the situation for $\mathrm{KI}$ where $\Delta t_{\mathrm{D}}$ is about 7 ps greater than $\Delta t_{\mathrm{L}}$ in the depicted region. This broadening results from the noncollinear propagation of the spatially extended $\left(x_{\mathrm{L}}, x_{\mathrm{P}}\right)$ pump pulses which causes an elongation of the temporal pulse overlap. The experimental half-width of $\Delta t_{\mathrm{D}} \simeq 12$ ps (Fig. 2) gives a pulse duration of $\Delta t_{\mathrm{L}} \simeq 5 \mathrm{ps}$. The correlation technique $\left[G\left(t_{\mathrm{D}}\right)\right.$ of (4)] leads to $\Delta t_{\mathrm{D}}=1.15 \Delta t_{\mathrm{L}}$ (dashed curve). Curve 2 is responsible for the situation of noncollinear four wave mixing in water $\left(l=2 \mathrm{~cm}, \varphi=10.68^{\circ}\right.$, $n_{\mathrm{L}}=1.3247, n_{\mathrm{P}}=1.33468, n_{\mathrm{S}}=1.34815, n_{g \mathrm{~L}}=1.33518$, $n_{g \mathrm{P}}=1.35783, \quad n_{g \mathrm{~S}}=1.39823, \quad \alpha_{\mathrm{L}}=0.172 \mathrm{~cm}^{-1}, \quad \alpha_{\mathrm{P}}$ $=3.2 \times 10^{-4} \mathrm{~cm}^{-1}, \alpha_{\mathrm{S}}=0.0023 \mathrm{~cm}^{-1}$ [13]). In earlier investigations on four-wave mixing in water [14] a half-width of $\Delta t_{\mathrm{D}} \simeq 6.5 \mathrm{ps}$ was found giving a pulse duration of $\Delta t_{\mathrm{L}} \simeq 5 \mathrm{ps}$.

The background intensity level $\delta_{\mathrm{L}}$ is only approximately equal to the energy conversion ratio $r\left(\left|t_{\mathrm{D}}\right|>6 \Delta t\right)$. The relation between $\delta_{\mathrm{L}}$ and $r\left(\left|t_{\mathrm{D}}\right|>6 \Delta t_{\mathrm{L}}\right)$ depends on the pulse duration $\Delta t_{\mathrm{L}}$ as shown in Fig. $4(\mathrm{KI}$, Curve 1; $\mathrm{H}_{2} \mathrm{O}$, Curve 2). The overlap of the pump pulses is reduced with decreasing pulse duration (group dispersion separates pulses). This fact causes a reduced energy conversion of the pulses (Fig. 3b, Curve $a$ for KI, Curve $b$ for $\mathrm{H}_{2} \mathrm{O}$ ) while the back- 


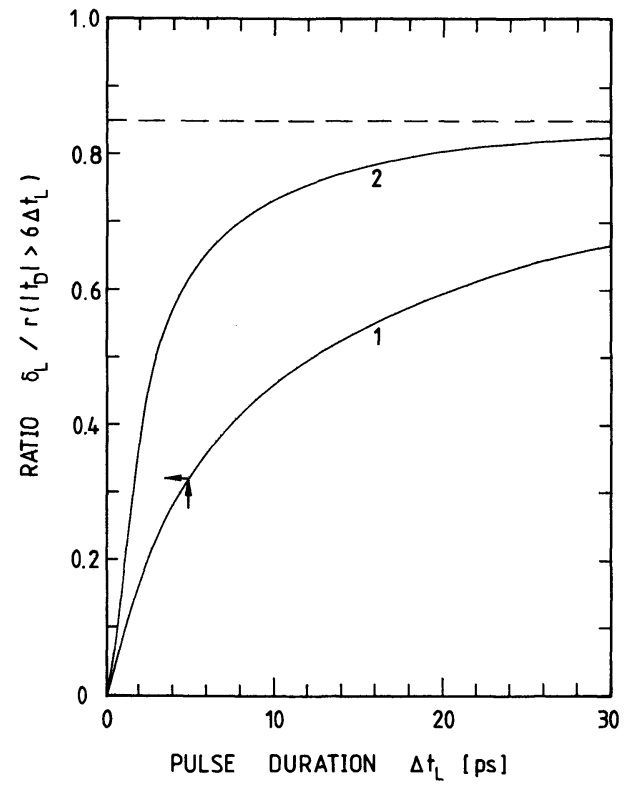

Fig. 4. Relation between background intensity level and energy conversion of background signal. Curve 1: interaction in $\mathrm{KI}$; Curve 2: interaction in $\mathrm{H}_{2} \mathrm{O}$; dashed curve, result of collinear interaction, (4). Pulse parameters as in Fig. 2

ground signal at frequency $v_{\mathrm{L}}$ interacts completely with the pump pulse at frequency $v_{\mathrm{p}}$. In our experiments it is $r\left(\left|t_{\mathrm{D}}\right|>6 \Delta t_{\mathrm{L}}\right) \simeq 1.5 \times 10^{-5}$ and the background intensity level is $\delta_{\mathrm{L}} \simeq 5 \times 10^{-6}$. The background level is rather constant over the investigated time region (Fig. 2). The correlation function analysis $[(4)$ dashed curve $]$ gives a ratio of $\delta_{\mathrm{L}} / r\left(\left|t_{\mathrm{D}}\right|>6 \Delta t\right)=0.85$ independent of the pulse duration $\Delta t_{\mathrm{L}}$.

The asymmetry of the laser pulses causes an asymmetry of the energy conversion versus delay time. The asymmetry of $r\left(t_{\mathrm{D}}\right)$ is lowered by the finite width $\Delta t_{\mathrm{p}}$ of the pump pulse at frequency $v_{\mathrm{P}}$ and the elongated pulse overlap by the noncollinear arrangement. The asymmetry ratio $A=\left(t_{d}-t_{r}\right) /\left(t_{d}+t_{r}\right)$ is plotted versus pulse duration $\Delta t_{\mathrm{L}}$ in Fig. 5. $t_{d}$ and $t_{r}$ are defined as decay time and rise time by $\eta\left(t_{\mathrm{D}}=-t_{d}<0\right)=\eta\left(t_{\mathrm{D}}=t_{r}>0\right)$ $=\eta\left(t_{\mathrm{D}}=0\right) / 1000$. In Fig. $5 \mathrm{a}$ the curves belong to fourwave mixing in $\mathrm{KI}(1, \kappa=4 ; 2, \kappa=1.6 ; 3, \kappa=0.6338)$ with a pulse duration ratio of $\Delta t_{\mathrm{L}} / \Delta t_{\mathrm{p}}=1.2$. The experimentally observed asymmetry $A \simeq 0.09$ indicates a pulse asymmetry parameter of $\kappa \simeq 1.6$. In case of idealized forward four-photon interaction, (4), the asymmetry ratio is only reduced by the finite width of $\Delta t_{\mathrm{P}}$ (dashed curves; $a, \kappa=4 ; b, \kappa=1.6$ ). The curves in Fig. $5 \mathrm{c}$ indicate the influence of the pulse duration ratio $\Delta t_{\mathrm{L}} / \Delta t_{\mathrm{P}}\left(1\right.$ and $a: \kappa=4 ; 2$ and $b: \kappa=1.6 ; \Delta t_{\mathrm{L}} / \Delta t_{\mathrm{P}}$ $=2^{1 / 2}$, medium KI). Figure $5 \mathrm{~b}$ shows the asymmetry for four-wave mixing in water $(1, \kappa=4 ; 2, \kappa=1.6$; $\left.\Delta t_{\mathrm{L}} / \Delta t_{\mathrm{P}}=1.2\right)$.

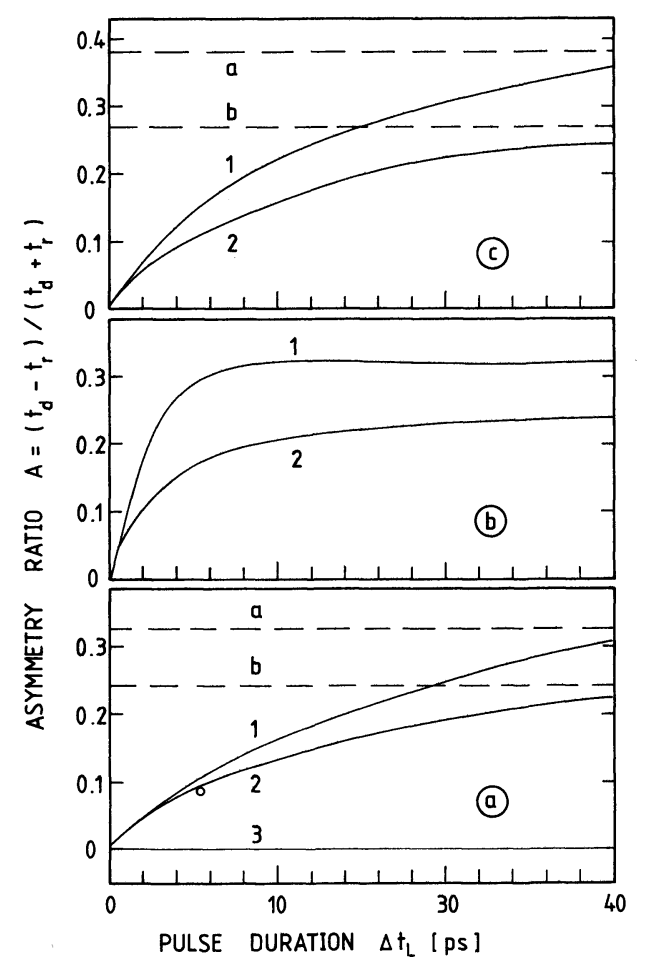

Fig. 5a-c. Asymmetry ratio of energy conversion curves versus pulse duration. (a) Interaction in $\mathrm{KI}$; pulse duration ratio $\Delta t_{\mathrm{L}} / \Delta t_{\mathrm{P}}=1.2$; (b) $\mathrm{H}_{2} \mathrm{O} ; \Delta t_{\mathrm{L}} / \Delta t_{\mathrm{P}}=1.2 ;$ (c) medium KI, $\Delta t_{\mathrm{L}} / \Delta t_{\mathrm{P}}=2^{1 / 2}$. Solid curves: $1, \kappa=4$ (pulse asymmetry $A_{\mathrm{L}}=0.73$, similar defined as $A$ ) $; 2$, $\kappa=1.6\left(A_{\mathrm{L}}=0.43\right) ; 3, \kappa=0.6338\left(A_{\mathrm{L}}=0\right)$. Dashed curves represent result of correlation functions (independent of substance). $a, \kappa=4 ; b$, $\kappa=1.6$

After the optimum overlap position $t_{\mathrm{D}}=0$ and the energy conversion $\eta\left(t_{\mathrm{D}}=0\right)$ have been determined, the measurement of the energy conversion at a fixed delay time $\bar{t}_{\mathrm{D}}$ allows the measurement of pulse durations in single shots. The pulse shape has to be assumed for these single shot measurements as it is necessary in the conventional two-photon fluorescence or secondharmonic correlation techniques.

The background energy content of the mode-locked laser may be reduced by passing the laser output through a saturable absorber. By putting a bleachable dye of small signal transmission $T_{0}=0.005$ (Eastman 9860) behind the laser amplifier, the background level decreased by a factor of 50 to $\delta_{\mathrm{L}} \simeq 10^{-7}$.

The lowest measurable background intensity level is approached when the light generation at frequency $v_{\mathrm{S}}$ by one of the pump pulses at either $v_{L}$ or $v_{\mathrm{P}}$ alone becomes comparable to that by four-wave mixing of background intensity at $v_{\mathrm{L}}$ with pump laser at $v_{\mathrm{p}}$. The fundamental laser at $v_{\mathrm{L}}$ generates light at $v_{\mathrm{S}}$ in forward direction by phase-mismatched third-harmonic generation. Curve 3 in Fig. 6 shows the energy conversion efficiency of third harmonic generation in forward 


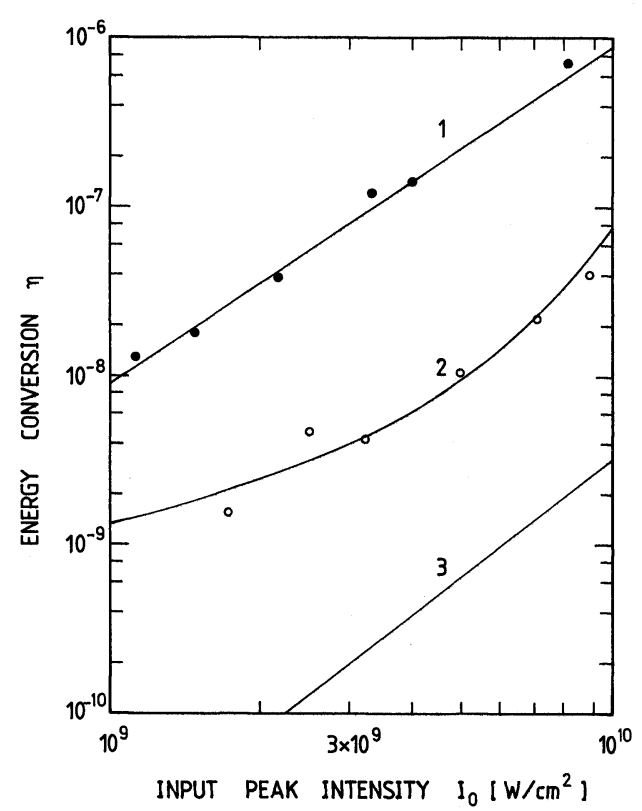

Fig. 6. Energy conversion by nonlinear optical processes in $\mathrm{KI}$ sample $(l=2 \mathrm{~cm})$. Curve 1 : four-photon frequency mixing $v_{\mathrm{P}}+v_{\mathrm{P}}$ $-v_{\mathrm{L}} \rightarrow v_{\mathrm{S}}$ of background pulses at $v_{\mathrm{L}}$ with pump pulse at $v_{\mathrm{P}}$. Closed circles, experimental points. Curve 2: parametric four-photon interaction $v_{\mathrm{P}}+v_{\mathrm{P}} \rightarrow v_{\mathrm{L}}+v_{\mathrm{S}}$ of pump pulse at frequency $v_{\mathrm{P}}$. Open circles, experimental data. Curve 3: phase-mismatched third-harmonic generation of pump pulse at frequency $v_{\mathrm{L}}$ in forward direction

direction for $\mathrm{KI}$ as nonlinear medium $(l=2 \mathrm{~cm}$, $\chi_{x x x x}^{(3)}\left(-v_{\mathrm{S}} ; v_{\mathrm{L}}, v_{\mathrm{L}}, v_{\mathrm{L}}\right)=4.8 \times 10^{-22} \mathrm{~m}^{2} / \mathrm{V}^{2}=4 \times 10^{-14}$ esu [13], $\Delta k=2.3 \times 10^{4} \mathrm{~cm}^{-1}$, using [Ref 19, Eq. (22)]). The generated third-harmonic light does not enter the photomultiplier (Fig. 1) and is negligibly small compared to the signal due to background pulse interaction at $v_{\mathrm{L}}$ with pump pulse at frequency $v_{\mathrm{P}}$ (Curve 1, full circles). The second-harmonic pump pulse generates light at frequency $v_{\mathbf{S}}$ by phase-matched noncollinear parametric four-photon interaction $v_{\mathbf{P}}$ $+v_{\mathbf{P}} \rightarrow v_{\mathbf{L}}+v_{\mathbf{S}}[19]$ (same phase-matching angle $\varphi$ ). The open circles indicate the measured energy conversion values $\eta=W_{\mathrm{S}} / W_{\mathrm{L}}=\alpha W_{\mathrm{S}} / W_{\mathrm{P}}\left(\alpha=W_{\mathrm{P}} / W_{\mathrm{L}} \simeq 0.2\right)$. The Curve 2 is calculated by use of [Ref. 19, Eqs. (11) and (13)] with $\chi_{x x x x}^{(3)}\left(-v_{\mathrm{S}} ; v_{\mathrm{P}}, v_{\mathrm{P}},-v_{\mathrm{L}}\right)=3 \chi_{x x x x}^{(3)}\left(-v_{\mathrm{S}}, v_{\mathrm{L}}, v_{\mathrm{L}}\right.$, $\left.v_{L}\right)=1.2 \times 10^{-13}$ esu [20], effective sample length $l_{\text {eff }}=1 \mathrm{~cm}$, width of the generated light $\Delta v=40 \mathrm{~cm}^{-1}$ (half-width of interference filter) and solid angle $\Delta \Omega=5 \times 10^{-4} \mathrm{sr}$ (adjusted to experimental points). In the intensity region around $I_{\mathrm{OP}} \simeq 2 \times 10^{9} \mathrm{~W} / \mathrm{cm}^{2}$ the parametric four-photon light is about a factor of 20 below the background signal level of fourphoton frequency mixing.

\section{Conclusions}

Noncollinear phase-matched four-photon frequency mixing was used to analyse the temporal behaviour of a mode-locked Nd-glass laser. The technique allows to measure the duration, shape, and background content of picosecond lasers with high accuracy. Pulse duration measurements in single shots are possible. Any transparent liquid or solid with reasonable electronic nonlinear susceptibility may be used as interaction medium. Media with small phase-matching angles (e.g. water) are preferable for the analysis of pulses with short duration $\left(\Delta t_{\mathrm{L}}<3 \mathrm{ps}\right)$.

Acknowledgements. The authors thank the "Deutsche Forschungsgemeinschaft" for financial support and the "Rechenzentrum" of the University for disposal of computer time.

\section{References}

1. D.J.Bradley, G.H.C.New: Proc. IEEE 62, 313 (1974)

2. P.W.Smith, M. A.Duguay, E.P.Ippen : Prog. Quantum Electr. 3, 107 (1974)

3. E.P.Ippen, C.V.Shank: In Ultrashort Light Pulses, ed. by S.L. Shapiro, Topics Appl. Phys. 18 (Springer, Berlin, Heidelberg, New York 1977) p. 83

4. J.Janszky, G.Conradi, R.N.Gyuzalian: Opt. Commun. 23, 293 (1977)

5. R.N.Gyuzalian, S.B.Sogomonian, Z.G.Horvath: Opt. Commun. 29, 239 (1979)

6. C. Kolmeder, W.Zinth, W. Kaiser: Opt. Commun. 30, 453 (1979)

7. R.Wyatt, E.E.Marinero: Appl. Phys. 25, 297 (1981)

8. J.Wiedmann, A.Penzkofer: Opt. Commun. 30, 107 (1979)

9. J.Wiedmann, A.Penzkofer: Opt. Commun. 25, 226 (1978)

10. G. Albrecht, A. Antonetti, G.Mourou: Opt. Commun. 40, 59 (1981)

11. A.Bimontas, R.Kupris, A.Piskarskas, V.Smil'gyavichyus, A.Stabinis: Sov. J. Quantum Electron. 8, 1475 (1978)

12. D.H. Auston: Appl. Phys. Lett. 18, 249 (1971)

13. A.Penzkofer, J.Schmailzl, H.Glas: Appl. Phys. B 29, 37 (1982)

14. A.Penzkofer, J.Kraus, J.Sperka: Opt. Commun. 37, 437 (1981)

15. R.C.Miller, G.D. Boyd, A. Savage: Appl. Phys. Lett. 6, 77 (1965)

16. J.Comly, E.Garmire: Appl. Phys. Lett. 12, 7 (1968)

17. W.H.Glenn: IEEE J. QE-5, 284 (1969)

18. S.A.Akhmanov, A.P.Sukhorukov, A.S.Chirkin: Sov. Phys. JETP 28, 748 (1969)

19. A.Penzkofer, W.Kaiser: Opt. Quantum Electron. 9, 315 (1977)

20. R.W.Terhune, P.D.Maker: In Lasers, Vol. 2, ed. by A.K.Levine (Marcel Dekker, New York 1967) Chap. 4, p. 295 